\title{
PSEUDO-EINSTEIN AND Q-FLAT METRICS WITH EIGENVALUE ESTIMATES ON CR-HYPERSURFACES
}

\author{
Jianguo $\mathrm{CaO}^{1}$ and Shu-Cheng $\mathrm{Chang}^{2}$
}

\begin{abstract}
.
In this paper, we will use the Kohn's $\bar{\partial}_{b}$-theory on CR-hypersurfaces to derive some new results in CR-geometry.

Main Theorem. Let $M^{2 n-1}$ be the smooth boundary of a bounded strongly pseudoconvex domain $\Omega$ in a complete Stein manifold $V^{2 n}$. Then (1) For $n \geq 3, M^{2 n-1}$ admits a pseudo-Einstein metric; (2) For $n \geq 2, M^{2 n-1}$ admits a Fefferman metric of zero CR Q-curvature; and (3) for a compact strictly pseudoconvex CR emendable 3-manifold $M^{3}$, its CR Paneitz operator $P$ is a closed operator.

There are examples of non-emendable strongly pseudoconvex CR manifold $M^{3}$, for which the corresponding $\bar{\partial}_{b}$-operator and Paneitz operators are not closed operators.
\end{abstract}

\section{Introduction}

In this paper, we study several questions, including the existence of $Q$-flat metrics, pseudo-Einstein metrics and the closedness of the CR Paneitz operators.

First, we will use an approach proposed by Fefferman and his school to prove that "the complete Kähler-Einstein $g_{\infty}$ on an open domain $\Omega$ induces a metric on $M=b \Omega$ with zero $\mathrm{CR} Q$-curvature, where $\Omega$ is a smooth, bounded strictly pseudo-convex domain in a Stein manifold $V^{2 n}$." To achieve this goal, we solve a $\partial \bar{\partial}$-Poincaré-LeLong equation via the $\bar{\partial}$-theory. Although this part does not produce new hard a-priori estimates, it is still valuable for other potential applications.

\footnotetext{
${ }^{1}$ Supported partially by NSF Grant DMS 0404558. The first named author is very grateful to National Center for Theoretical Sciences at National Tsinghua University for its warm hospitality.

${ }^{2}$ Supported partially by the NSC of Taiwan
} 
The second purpose is to prove the existence of pseudo-Einstein metrics on strictly pseudo-convex $C R$-hypersurface of real dimension $\geq 5$ through solving the $\bar{\partial}_{b}$ Poincaré-LeLong equations.

The last part of our paper is to study the closedness of CR Paneitz operator, which is a fourth-order differential operator. It is known that the positivity of CR Paneitz operator is related to the deformation of $Q$-curvatures under the conformal change of metrics on Riemannian manifold $M^{m}$. In particular, the positivity of CR Paneitz operator is also related to the lower bound of the first eigenvalue of subLaplace on a $C R$ manifold $M^{3}$, see [CC], [CCC] and [LL]. It will be shown that, if $M^{3}=b \Omega^{4}$ is the smooth boundary of bounded strictly pseudo-convex domain $\Omega$ in a Stein manifold $V^{4}$, then its CR-Paneitz operator on $M^{3}$ is closed, for any metric on $M^{3}$.

Main Theorem. Let $M^{2 n-1}$ be the smooth boundary of a bounded strongly pseudoconvex domain $\Omega$ in a complete Stein manifold $V^{2 n}$. Then

(1) For $n \geq 2, M^{2 n-1}$ admits a metric of zero $C R$ Q-curvature;

(2) For $n \geq 3, M^{2 n-1}$ admits a pseudo-Einstein metric;

(3) In addition, for a compact strictly pseudoconvex CR emendable 3-manifold $M^{3}$, its CR Paneitz operator $P$ is a closed operator.

Earlier work in this direction for the case of $V^{2 n}=\mathbb{C}^{n}$ can be found in [L2], [FH] and [GG]. In a very recent paper [LL], Li and Luk obtained an explicit formula for Webster's pseudo-Ricci curvature on real hypersurfaces in $\mathbb{C}^{n}$. Thus, their result could lead another proof of Cheng-Yau's result ([CY]) and Mok-Yau's theorem [MY], which will be used in Section 2 below.

Among other things, we introduce some new methods to handle pseudo-Einstein metric and Paneitz operators in this paper. For example, we use the closeness of $\bar{\partial}_{b}$ and $\bar{\partial}_{b}^{*}$ operators provided by Kohn's theory, in order to complete the proof. When $\operatorname{dim}_{\mathbb{R}}[M]=3$, we decompose the Paneitz operator $P$ as a product of closed 
operators. Thus, the closed property of $P$ will follow immediately, see Lemma 1.4 and Section 4 below.

\section{Preliminary Results}

It is well-known that the real Laplace $\triangle$ on a Kähler manifold $V^{2 n}$ satisfies

$$
\triangle=2 \square=2 \bar{\square},
$$

where $\square=\bar{\partial} \bar{\partial}^{*}+\bar{\partial}^{*} \bar{\partial}$ is the complex Laplace operator. However, it may happen that $\triangle_{b} \neq 2 \square_{b}$ in some cases. Let us recall the notions of $\triangle_{b}$ and $\square_{b}$.

Since $M^{2 n-1}=b V^{2 n}$ has odd real dimension, it is a Cauchy-Riemann manifold. The $\bar{\partial}_{b}$ operator induces a sub-elliptic operator

$$
\square_{b}=\bar{\partial}_{b}^{*} \bar{\partial}_{b}+\bar{\partial}_{b} \bar{\partial}_{b}^{*}
$$

acting on $L_{(p, q)}^{2}(M)$. Similarly, there is a real sub-Laplace operator, which can be viewed as partial trace of the hessian operator (or can be viewed a sum of the squares of $(2 n-2)$ vectors):

$$
\left.\triangle_{b} u\right|_{z}=\left.\sum_{k=1}^{2 n-2}\left\langle\nabla_{e_{k}}\left(\nabla^{b} u\right), e_{k}\right\rangle\right|_{z}
$$

where $e_{2 n}$ is the outward real unit normal vector of $\Omega$ along boundary $M=$ $b \Omega, e_{2 j}=J e_{2 j-1}$ for $j=1, \ldots, n, z \in M, J$ is the complex structure of $V^{2 n}$, $\left\{e_{1}, e_{2}, \cdots, e_{2 n-3}, e_{2 n-2}, e_{2 n-1}, e_{2 n}\right\}$ is an orthonormal basis of $\left[T_{z}(V)\right]_{\mathbb{R}}$ and

$$
\nabla^{b} u=\sum_{k=1}^{2 n-2} d u\left(e_{k}\right) e_{k}
$$

When $\Omega$ has the strongly pseudo-convex boundary in a Stein manifold $V^{2 n}$ with $n=2$, it has been observed that

$$
\square_{b} u=\frac{1}{2}\left[\triangle_{b} u+\sqrt{-1} T u\right]
$$


for all $u \in L^{2}\left(M^{3}\right)$, where $T=\lambda e_{3}$ is the Reeb vector of the CR 3-manifold $M^{3}$ for some real valued function $\lambda$, see [L1, p414].

The operator $\square_{b}$ is a Lewy type operator, which may not be locally solvable.

If the Reeb vector $T$ induces an infinitesimal pseudo-conformal with respect to the Tanaka-Webster metric, then the torsion of $M^{3}$ is zero, see [Web, p33]. In this case, the operator $\square_{b}$ is related to the so-called CR Paneitz operator $P$, where $P$ is given by

$$
P u=\triangle_{b}^{2} u+T^{2} u=4 \square_{b} \square_{b} u,
$$

for $u \in L^{2}\left(M^{3}\right)$. More generally, if $M^{3}$ has torsion free in the sense of Tanaka (cf. [Ta1-2] [Web]), then (1.2) holds.

The eigenvalues of the Paneitz operator and CR Paneitz operators have been considered various authors $([\mathrm{Ch}],[\mathrm{CC}])$. The eigenvalue estimate plays an important role to the study of the so-called Q-curvature flow, see [Br] [CCC].

Definition 1.1. (1) The CR-Paneitz operator $P: L^{2}\left(M^{3}\right) \rightarrow L^{2}\left(M^{3}\right)$ is called essentially positive, if there is a positive constant $\lambda_{1}>0$ such that

$$
\langle P u, u\rangle\left\|\geq \lambda_{1}\right\| u \|^{2}
$$

for all $u \perp k e r(P)$.

(2) The operator $\mathcal{F}: L_{(p, q)}^{2}(M) \rightarrow L_{(p, q)}^{2}(M)$ is said to have positive spectrum gap at 0 (or is said to be a closed operator) if there is a positive constant $\lambda_{p, q}>0$ such that

$$
\|\mathcal{F} u\| \geq \lambda_{p, q}\|u\|
$$

for all $u \perp\left[L_{(p, q)}^{2}(M) \cap \operatorname{ker}(\mathcal{F})\right]$.

(3) A smooth function $f: U_{\varepsilon}(M) \rightarrow \mathbb{R}$ is called a defining function of $M$ if $f^{-1}(0)=M$ and if 0 is not a critical value of $f$, where $U_{\varepsilon}(M) \subsetneq V^{2 n}$ is a neighborhood of $M$ in a Stein manifold $V^{2 n}$.

(4) Let $\theta$ be a contact 1 -form of $M^{2 n-1}$ and $J: \operatorname{ker} \theta \rightarrow \operatorname{ker} \theta$ be the almost complex structure on the CR-distribution $\operatorname{ker} \theta$ such that $J^{2} \vec{v}=-\vec{v}$ for all $\vec{v} \in \operatorname{ker} \theta$. 
In what follows, we always let

$$
\left[T^{(1,0)}(M) \oplus T^{(0,1)}(M)\right]=[\operatorname{ker} \theta] \bigotimes_{\mathbb{R}} \mathbb{C}
$$

(5) A CR manifold $M^{2 n-1}$ is said to have transverse symmetry or torsion-free if it admits a CR Reeb vector field $\xi$ such that $\xi \notin \operatorname{ker} \theta$ with

$$
\mathcal{L}_{\xi} J=0
$$

where $\mathcal{L}$ is the Lie derivative and $J$ is the complex structure of $\left[T^{(1,0)}(M) \oplus T^{(0,1)}(M)\right]$.

If $\xi$ is the real part of a holomorphic vector filed $\tilde{X}$ on a neighborhood $U_{\varepsilon}(M)$ of $M$, then $\xi$ induces an automorphism on $U_{\varepsilon}(M)$. Any real part $\xi$ of a holomorphic vector filed restricted to $M$ induces a CR-automorphism of $M$.

In the Hörmand-Kohn $L^{2}$-theory and the Kohn-Rossi theory, the essential spectrum of $\square$ and $\square_{b}$ have been extensively investigated.

A smooth $(p, q)$-form $u$ on $\Omega$ with $q \geq 1$ is said to satisfy the $\bar{\partial}$-Neumann boundary condition if

$$
\left.u\left((\bar{\partial} \rho)_{\#}, \ldots\right)\right|_{z}=0
$$

for all $z \in M=b \Omega$, where $(\bar{\partial} \rho)_{\#}$ is the complex normal vector field of type $(0,1)$ along the boundary $M^{2 n-1}$.

Theorem 1.2. ([CS], [CaWS]) Let $\Omega$ be a bounded domain with smooth pseudoconvex boundary $M$ in a complete Hermitian manifold $V^{2 n}$. Suppose that $V^{2 n}$ is either a Stein manifold or $\mathbb{C} P^{n}$. Then the complex Laplace operator $\square$ is

(1) positive for on $L_{(p, q)}^{2}(\Omega)$ with $(n-1) \geq q \geq 1$; and

(2) essentially positive on $L_{(p, 0)}^{2}(\Omega)$ and $L_{(p, n)}^{2}(\Omega)$

with respect to $\bar{\partial}-$ Neumann boundary condition on $M=b \Omega$.

Moreover, for any Hermitian metric on $\Omega$, the operator $\square$ is essentially positive on $\Omega$ with respect to $\bar{\partial}-N$ eumann boundary condition on $M$. 
For the $L^{2}$ estimates of $\square$, the domains $\Omega$ in Theorem 1.2 are not necessarily strictly pseudo-convex. However, for estimates of $\square_{b}$ on the boundary $M^{2 n-1}$ of $\Omega$, we need extra assumptions on $M^{2 n-1}$.

The dual of $\bar{\partial}$-Neumann problem is the so-called $\bar{\partial}$-Cauchy problem. A $(p, q)$-form $u$ is said to satisfy the Cauchy boundary condition on $M=b \Omega$ if

$$
\left.u(\xi, \ldots)\right|_{z}=0
$$

for all $\xi \in T_{z}^{(0,1)}(M)$ and $z \in M$. If a $\bar{\partial}$-closed form $f \in C_{(p, q+1)}^{\infty}(\Omega)$ with a compact support in $\Omega$, then one consider to solve $\bar{\partial} u=f$ such that $u$ has a compact support in $\Omega$ as well. Solving $\bar{\partial} u=f$ with compact support is related to the $\bar{\partial}$-extension problem, via the Kohn-Rossi theory. Using the solution to the $\bar{\partial}$-extension problem and Theorem 1.2, we are able to solve $\bar{\partial}_{b} u=f$ on a special class of CR-manifolds:

Theorem 1.3. ([CS], [CaSW]) Let $\Omega$ be a bounded Hermitian manifold with a smooth pseudo-convex boundary $M$. Suppose that one of the following conditions holds:

(1) $\Omega$ is a domain of a complete Stein manifold $V^{2 n}$;

(2) $\Omega \subset \mathbb{C} P^{n}$, and $M=b \Omega$ admits a pluri-subharmonic defining function.

Then the $\bar{\partial}$-Cauchy boundary problem is solvable on $\Omega$. Furthermore, (1) $\bar{\partial}_{b}$ operator is closed; and (2) the operator $\square_{b}: L_{(p, q)}^{2}(M) \rightarrow L_{(p, q)}^{2}(M)$ is positive for $1 \leq q \leq n-2$ and essentially positive for $q=0$ or $q=n-1$.

When $M=b \Omega$ is strongly pseudo-convex, it is well-known that $M$ admits a pluri-subharmonic defining function, see $[\mathrm{DF}]$.

If $\mathcal{L}: H_{1} \rightarrow H_{2}$ is a linear operator, we let $\operatorname{Dom}(\mathcal{L})$ be its domain and $\mathcal{R}(\mathcal{L})$ be its range. If $A \subset H$ is a subset of a Hilbert space $H$, the closure of $A$ in $H$ is denoted by $\bar{A}$.

We begin with an elementary but useful criterion for closed operators.

Lemma 1.4. ([CS, p60] or [Hö1-2]) Let $\mathcal{L}: H_{1} \rightarrow H_{2}$ be a linear, closed, densely 
defined operator from the Hilbert space $\mathrm{H}_{1}$ to another Hilbert space $\mathrm{H}_{2}$. The following conditions on $\mathcal{L}$ are equivalent:

(1) The range $\mathcal{R}(\mathcal{L})$ of $\mathcal{L}$ is closed;

(2) There is a constant $C$ such that

$$
\|f\|_{1} \leq C\|\mathcal{L} f\|_{2}
$$

for all $f \in \operatorname{Dom}(\mathcal{L}) \cap \mathcal{R}\left(\mathcal{L}^{*}\right)$;

(3) The range $\mathcal{R}\left(\mathcal{L}^{*}\right)$ of $\mathcal{L}^{*}$ is closed;

(4) There is a constant $C$ such that

$$
\|f\|_{2} \leq C\left\|\mathcal{L}^{*} f\right\|_{1}
$$

for all $f \in \operatorname{Dom}\left(\mathcal{L}^{*}\right) \cap \mathcal{R}(\mathcal{L})$.

\section{The EXIStence of CR $Q$-Flat Metrics on STRICTLY} PSEUdo-CONVEX CR-HypersurfaCes in A Stein MANIFOld

In this section, we first recall an existence result of $\mathrm{CR} Q$-flat metrics on CRhypersurfaces in Euclidean space $\mathbb{C}^{n}$ due to Fefferman and others. Afterwards, we will extend such a result to $C R$-hypersurfaces in an arbitrary Stein Manifold $V^{2 n}$. One of our key steps is to use the $\bar{\partial}$-theory to introduce the generalized Fefferman's functional $u \rightarrow \hat{J}(u)$, which is independent of the choice of local holomorphic coordinates, see (2.5) below.

\section{2.a. A sufficient condition for existence of $Q$-flat metrics on real hyper- surfaces.}

Let us recall a sufficient condition for existence of $Q$-flat metrics on real hypersurfaces, which were derived by Fefferman and others.

Proposition 2.0. ([FG1-2], $[G G])$ Let $\Omega \subset \mathbb{C}^{n}$ be a compact domain with smooth boundary $M^{2 n-1}=b \Omega$ in the complex Euclidean space $\mathbb{C}^{n}$. Suppose $\Sigma^{2 n}$ is an unit 
circle bundle defined on a CR-hypersurface $M^{2 n-1}$ and suppose that $\Sigma^{2 n}$ admits an $S^{1}$-invariant Einstein-Lorentz metric $g_{u}^{+}=\left.i \partial \bar{\partial} H_{u}\right|_{\Sigma^{2 n}}$ defined as below. Then $M^{2 n-1}$ admits a metric of zero $C R Q$-metric.

We now provide a description of the metric $g_{u}^{+}$stated in Proposition 2.0, which will be used for any real hypersurface $M^{2 n-1}$ in a Stein manifold $V^{2 n}$ as well.

Let $K^{*}$ be the canonical bundle of $V^{2 n}$ restrict to $M$ and let $\Sigma^{2 n}=K^{*} / \mathbb{R}^{+}$be the unit circle bundle of $K^{*}$. Thus there is a fiberation

$$
S^{1} \rightarrow \Sigma^{2 n} \rightarrow M^{2 n-1}
$$

and $\operatorname{dim}_{\mathbb{R}}\left(\Sigma^{2 n}\right)=2 n$.

We may assume that $\Omega \subset V^{2 n}$ is an open strictly pseudo-convex domain with compact smooth boundary $M^{2 n-1}=b \Omega$. Suppose that $\hat{u}$ is a defining function of $M^{2 n-1}$. For example, we can choose $\hat{u}$ as a signed distance function form $M$ :

$$
\hat{u}(z)=\left\{\begin{array}{l}
-d(z, M), \quad \text { if } \quad z \in \Omega \\
d(z, M), \quad \text { if } \quad z \notin \Omega
\end{array}\right.
$$

Any other defining function $u$ can be expressed as

$$
u(z)=e^{\eta} \hat{u}
$$

for some real valued function $\eta$.

The contact structure on $M$ is an 1-form given by

$$
\theta_{u}(\xi)=d u(J \xi)
$$

for all $\xi \in[T(M)]_{\mathbb{R}}$, where $J$ is the complex structure of $V^{2 n}$.

There are two types of metrics which we will use. The first one is the Cheng-Yau metric on $\Omega$; and the second one is introduced by Fefferman on a line bundle over $b \Omega$. 
Let us first consider complete Kähler metrics on an open domain $\Omega$. Suppose that

$$
\omega_{u}=i \partial \bar{\partial}\left[\log \left(-\frac{1}{u}\right)\right]
$$

is a Kähler form on $\Omega$. Such a Kähler form $\omega_{u}$ corresponds to a Kähler metric

$$
g_{u}(X, Y)=\omega_{u}(X, J Y)=i \partial \bar{\partial}\left[\log \left(-\frac{1}{u}\right)\right](X, J Y)
$$

where $J$ is the complex structure of $\Omega$.

Secondly, Fefferman and his school considered a class of Lorentz metrics on canonical bundle on $K^{*}$ mentioned above.

We will use an extrinsic way to define such metrics, along the line described in a new book [DT, p150]. Suppose that $\Lambda_{(n, 0)}\left(V^{2 n}\right)$ be the canonical line bundle of open domain $V^{2 n}$. Clearly, $\mathcal{L}_{V^{2 n}}=\Lambda_{(n, 0)}\left(V^{2 n}\right)$ is a complex manifold of complex dimension $(n+1)$.

When $\xi$ is a cross-section of $\mathcal{L}_{V^{2 n}}$ over $V^{2 n}$, the norm $|\xi|_{g_{u}}$ induced by $g_{u}$ is well-defined. We further define

$$
H_{u}(z, \xi)=|\xi|_{g_{u}}^{\frac{2}{n+1}} u(z)
$$

There is an $(1,1)$-form defined on $\mathcal{L}_{V^{2 n}}$ given by $i \partial \bar{\partial} H_{u}$.

Similarly, there is a Hermitian form

$$
G_{u}(\tilde{X}, \tilde{Y})=i \partial \bar{\partial} H_{u}(\tilde{X}, \tilde{J} \tilde{Y})
$$

where $\tilde{J}$ is the complex structure of line bundle $\mathcal{L}_{V^{2 n}}$. The Hermitian form $G_{u}$ is not necessarily positive definite on the complex manifold $\mathcal{L}_{V^{2 n}}$.

We now consider a subset

$$
\Sigma^{2 n}=\left\{(z, \xi) \in \mathcal{L}_{V^{2 n}} \quad|\quad z \in b \Omega,| \xi \mid=1\right\}
$$

where $\Omega$ is an open, bounded and strictly pseudo-convex domain in $V^{2 n}$. 
Finally, when $i \partial \bar{\partial} u>0$ on $M=b \Omega$, we consider

$$
g_{u}^{+}=\left.G_{u}\right|_{\Sigma^{2 n}} .
$$

It was shown that $g_{u}^{+}$is a Lorentz metric on $\Sigma^{2 n}$. Clearly, $\Sigma^{2 n}$ is diffeomorphic to the unit circle bundle $K^{*}$ mentioned above.

We remark that the function $u=0$ vanishes on $M^{2 n-1}$. The leading term of the metric $g_{u}^{+}$is

$$
i \partial \bar{\partial} u \text {. }
$$

In $[\mathrm{FH}]$, Fefferman and Hirachi studied the so-called $Q$-curvature of $C R$-manifold $M^{3}$ :

$$
Q_{\theta_{u}}^{C R}=\frac{4}{3}\left(\Delta_{b} R-2 \operatorname{Im} \nabla^{\alpha} \nabla^{\beta} A_{\alpha \beta}\right),
$$

where $R$ is the Tanaka-Webster scalar curvature, $A$ is the torsion, $\Delta_{b}$ is the subLaplacian computed in terms of the contact 1-form $\theta_{u}$ and $\theta_{u}(\xi)=d u(J \xi)$ for all $\xi \in T(M)$.

For higher dimensional manifolds, the $Q$-curvatures of higher order have been studied in $[\mathrm{FH}]$ and $[\mathrm{GG}]$.

The notations above will be used in the next two sub-sections.

\section{2.b. Relations between the Fefferman's Lorentz metric and the Cheng- Yau's Kähler-Einstein metric.}

In this sub-section, we illustrate a strategy to obtain the existence of $Q$-flat metrics on real hypersurfaces in $\mathbb{C}^{n}$.

Let us now recall a result obtained by Fefferman and his school.

Proposition 2.1. ([FG1, Chapter III]) Let $\Omega \subset V^{2 n}, M=b \Omega \subset \mathbb{C}^{n}, u=\hat{u} e^{\eta}$ and $\left\{g_{u}, g_{u}^{+}\right\}$be as above. If the Cheng-Yau metric $g_{u}$ is a complete Kähler-Einstein on $\Omega$, then the Lorentz metric $g_{u}^{+}$is Einstein on $\Sigma^{2 n}$.

Here is a direct application of Propositions 2.0-2.1. 
Corollary 2.2. ([FH], $[G G])$ Let $\Omega \subset \mathbb{C}^{2 n}$ be an open strictly pseudo-convex domain with compact closure and let $M^{2 n-1}=b \Omega$ be its boundary. Then $M$ admits a metric of zero CR Q-curvature.

Proposition 2.1 and Corollary 2.2 were stated for strictly pseudo-convex and bounded domain $\Omega$ in $\mathbb{C}^{n}$. We would like to extend these results to any strictly pseudo-convex and bounded domain $\Omega$ in a Stein manifold $V^{2 n}$.

\section{2.c. Compact smooth real hypersurfaces in a Stein manifold.}

Our goal of this section is to verify the following theorem.

Proposition 2.3. Let $\Omega$ be a bounded, open and strictly pseudo-convex domain with a smooth boundary in a Stein manifold $V^{2 n}$. If the metric $g_{u}$ above is a complete Kähler-Einstein metric on $\Omega$, then $g_{u}$ induces a metric $\tilde{g}_{u}^{\infty}$ on $M=b \Omega$ with zero $C R Q$-curvature.

Proof. Since $V^{2 n}$ is Stein, we may assume that $V^{2 n} \subset \mathbb{C}^{m}$ is a complete submanifold of $\mathbb{C}^{m}$, for sufficiently large $m$. Let $\hat{g}$ be induced metric on $\Omega \subset V^{2 n} \subset \mathbb{C}^{m}$. For each local holomorphic coordinate system $\left\{\left(z_{1}, \ldots, z_{n}\right)\right\}$ of $\Omega$, the Ricci tensor $\hat{R} i c$ of $\hat{g}$ is given by

$$
\hat{R} i c=-i \partial \bar{\partial} \log \left[\operatorname{det} \hat{g}_{i \bar{j}}\right] .
$$

It is clear that $\hat{R} i c$ is well-defined and independent of the choice of local holomorphic coordinate system $\left\{\left(z_{1}, \ldots, z_{n}\right)\right\}$. Moreover, $\hat{R} i c$ is a closed $(1,1)$-form on $\Omega$. In what follows, we first would like to solve Poincare-Lelong equation $i \partial \bar{\partial} f=\hat{R} i c$.

For this purpose, we recall a theorem of Dolbeault:

$$
H^{(1,1)}(\Omega)=H^{(0,1)}\left(\Omega,\left.\mathcal{O}\right|_{\Omega}\right)
$$

where $\left.\mathcal{O}\right|_{\Omega}$ is the bundle of holomorphic $(1,0)$-forms.

Since $\Omega$ is strictly pseudo-convex and bounded domain in a Stein manifold $V^{2 n}$, by a theorem of Andreotti and Vesentini $[\mathrm{AV}]$, we have

$$
H^{(0,1)}\left(\Omega,\left.\mathcal{O}\right|_{\Omega}\right)=0 .
$$


In fact, Proposition A.4 of [CaWS, p218] is also applicable for $(0, \mathrm{q})$-forms with values in $\left.\mathcal{O}\right|_{\Omega}$. Thus, $H^{(1,1)}(\Omega)=H^{(0,1)}\left(\Omega,\left.\mathcal{O}\right|_{\Omega}\right)=0$. Professor Siu also handled similar formula with values in a vector bundle $E$, although the weighted functions were not discussed there (cf. [Siu, Chapters 2-3]). Hence, the first Chern class $c_{1}\left(\left.\mathcal{O}\right|_{\Omega}\right)=0$. Recall that, by Chern-Weil theory, the co-homology class $c_{1}\left(\left.\mathcal{O}\right|_{\Omega}\right)$ is independent of the choices of affine connections, (cf. [Mi]). Therefore, $c_{1}\left(\left.\mathcal{O}\right|_{\Omega}\right)=0$ implies that the Chern-Weil form $\hat{R} i c$ is $d$-exact on $\Omega$.

Therefore, we have $\hat{R} i c=d \beta$ for some 1-form $\beta$. Let us consider the decomposition of $\beta=\beta^{(1,0)}+\beta^{(0,1)}$, where $\beta^{(0,1)}$ is the $(0,1)$-component of $\beta$. If $\hat{R} i c=d \beta$ and if $\beta=\beta^{(0,1)}+\beta^{(1,0)}$, then $\bar{\partial} \beta^{(0,1)}=0$, where we used the fact that $\hat{R} i c$ is an $(1,1)$-form. Choosing $f$ with $\bar{\partial} f=i \beta^{(0,1)}$, we get a solution $i \partial \bar{\partial} f=\hat{R} i c$.

Recall that $\hat{R i c}$ is real valued. Replacing $f$ by $\operatorname{Re}\{f\}$ if needed, we conclude that the Poincare-Lelong equation

$$
i \partial \bar{\partial} f=\hat{R} i c=-i \partial \bar{\partial} \log \left[\operatorname{det} \hat{g}_{i \bar{j}}\right]
$$

has a smooth real-valued solution $f$ on $\Omega \cup b \Omega$. Such a solution $f$ is unique up to adding a pluri-subharmonic function. If we require that $f$ has the smallest $L^{2}(\Omega)$ norm, then such a solution is unique, see Chapters 4-5 of [CS]. Such a solution $f$ is called a Ricci potential of $\hat{g}$.

Following Fefferman [F2], we consider

$$
\hat{J}(u)=(-1)^{n} e^{-f} \frac{1}{\operatorname{det} \hat{g}_{i \bar{j}}} \operatorname{det}\left(\begin{array}{cc}
u & u_{\bar{j}} \\
u_{i} & u_{i \bar{j}}
\end{array}\right)
$$

where $f$ is the Ricci potential of $\hat{g}$ as above, $u_{i}=\frac{\partial u}{\partial z_{j}}, u_{i \bar{j}}=\frac{\partial^{2} u}{\partial z_{i} \partial \bar{z}_{j}}$ and $\left\{z_{1}, \ldots, z_{n}\right\}$ is a local holomorphic frame.

When $\Omega \subset \mathbb{C}^{n}$, we choose the standard coordinate system. Thus, in this case, $\operatorname{det} \hat{g}_{i \bar{j}}=1$ and we can choose $f=0$. Therefore, our definition coincides with Fefferman's definition for the case of $\Omega \subset \mathbb{C}^{n}$, see [F2] and [CY]. 
A calculation similar to [CY, p508] further shows that the metric $g_{u}$ is KählerEinstein of negative curvature $-(n+1)$ if

$$
\frac{\operatorname{det} \varphi_{i \bar{j}}}{\operatorname{det} \hat{g}_{i \bar{j}}}=e^{f} e^{(n+1) \varphi}
$$

holds, where $\varphi=\log \left(-\frac{1}{u}\right)$.

A further calculation shows that the above equation holds if and only if

$$
\left.\hat{J}(u)\right|_{z} \equiv 1
$$

holds for all $z \in \Omega$.

It is known that if $\left.\hat{J}(u)\right|_{z} \equiv 1$ in $\Omega$, then $M=b \Omega$ has zero CR Q-curvature, see [FH, Chapter 3]. This completes the proof of Proposition 2.3.

Corollary 2.4. Suppose that $\Omega \subset V^{2 n}$ be a bounded, open and strictly pseudoconvex domain with smooth boundary in a Stein manifold $V^{2 n}$ with $n \geq 2$. Then its boundary $M^{2 n-1}=b \Omega$ admits a metric of zero $Q$-curvature.

Proof. By Proposition 2.3, it remains to verify that there is a complete KählerEinstein metric $g_{u}$ on $\Omega$. The existence of such a complete Kähler-Einstein metric $g_{u}$ is provided by Mok-Yau in [MY, p52]. In fact, Mok and Yau found desired solutions $u=e^{\eta} \hat{u}$ and $\varphi=\log \left(-\frac{1}{u}\right)$ satisfying $\frac{\operatorname{det} \varphi_{i \bar{j}}}{\operatorname{det} \hat{g}_{i \bar{j}}}=e^{f} e^{(n+1) \varphi}$.

\section{Existence of Pseudo-Einstein metrics on}

\section{CR-HYPERSURFACES OF REAL DIMENSION $\geq 5$}

In this section, we discuss the existence of pseudo-Einstein metrics on CRhypersurfaces of real dimension $\geq 5$. A metric $g$ defined on a $C R$-manifold $M^{2 n-1}$ is said to be pseudo-Einstein (or partially Einstein) if its Ricci tensor satisfies

$$
\left.\operatorname{Ric}_{g}(X, Y)\right|_{z}=\left.\lambda g(X, Y)\right|_{z}
$$

for some constant $\lambda=\lambda(z)$ and for all real vectors $\{X, Y\}$ in the $C R$-distribution $\left.\operatorname{ker}(\theta)\right|_{z}$, where $\theta$ is the contact form of $M^{2 n-1}$. 
One of our new contributions in this section is to use the $\bar{\partial}_{b}$-theory to solve boundary version of Poincaré-Lelong equation related to the partially Einstein equation, see Proposition 3.4 and Corollary 3.5 below.

When $\operatorname{dim}_{\mathbb{R}}\left[M^{2 n-1}\right]=3$, any metric $g$ on $M^{3}$ is pseudo-Einstein (i.e., partially Einstein). Therefore, we only consider the case of $\operatorname{dim}_{\mathbb{R}}\left[M^{2 n-1}\right] \geq 5$.

We emphasize that a pseudo-Einstein metric $g$ on $M^{2 n-1}$ is not necessarily Einstein. The pseudo-Einstein condition puts no restriction on its Ricci curvature in the directions which are transversal to $C R$-distribution. It might happen that

$$
\operatorname{Ric}_{g}(Z, Y) \neq \lambda g(Z, Y)
$$

for some transversal vector $Z \perp k e r(\theta)$.

In [L2], Lee already showed that, if a compact strongly pseudo-convex CRmanifold $M^{2 n-1}$ admits a closed, nowhere vanishing $(n, 0)$-form, then $M^{2 n-1}$ admits a pseudo-Einstein metric. In particular, if $M=b \Omega$ and $\Omega \subset \mathbb{C}^{n}$, then $M$ admits a pseudo-Einstein structure.

We make extra observations to extend Lee's result to the case of $\Omega \subset V^{2 n}$ for any Stein manifold $V^{2 n}$. The new ingredient of our approach will use the fact that the Chern curvature forms $\Theta$ are type of $(1,1)$ for Lorentz-Kähler metrics.

In addition, we will use Kohn's $\bar{\partial}_{b}$-theory to solve the boundary version of Poincare-Lelong equation

$$
i \partial_{b} \bar{\partial}_{b} f=\Theta
$$

for any $\bar{\partial}_{b}$-closed $(1,1)$-form $\Theta$.

The equation (3.1) above is related to the existence of pseudo-Einstein metrics, as described in [L2, p173]. Such an equation was previously studied in [CaWS] for other purposes.

It is well-known that, for any function $u$, one has

$$
\left(d^{c} u\right)(\xi)=(d u)(J \xi) \text { and } d d^{c} u=i \partial \bar{\partial} u \text {. }
$$

We begin with an elementary observation. 
Lemma 3.1. Let $\hat{u}$ be a defining function of $M=b \Omega$. Suppose that $\Omega \subset V^{2 n}$ is a strictly pseudoconvex bounded domain in a Stein manifold. Then

(1) There is another defining function $u=e^{\varphi} \hat{u}$ such that $u$ is a strictly pluri-

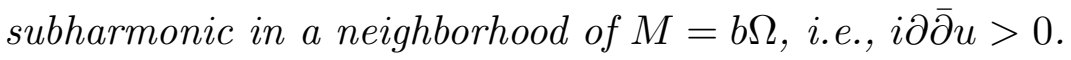

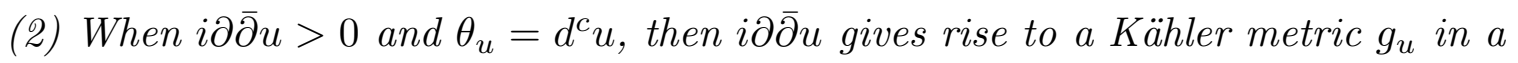
neighborhood of $M$.

(3) If $u=e^{\varphi} \hat{u}, \theta=d^{c} u$ and $\hat{\theta}=d^{c} \hat{u}$, then one has

$$
\theta=e^{\varphi} \hat{\theta} \text { on } M
$$

Proof. Assertion (1) was stated in Theorem 3.4.4 of [CS, p45-46].

The verification of Assertions (2)-(3) is straightforward.

Proposition 3.2. Let $\Omega$ be a bounded, strictly pseudo-convex domain with a smooth boundary $M=b \Omega$ in a Stein manifold $V^{2 n}$, let $\mathcal{O}$ be the holomorphic $(1,0)$-form bundle of $V^{2 n}$, and let $K^{*}$ be the canonical line bundle of $V^{2 n}$. Suppose that $\operatorname{dim}_{\mathbb{R}}\left[V^{2 n}\right]=2 n \geq 6$. Then the following is true.

(1) The first Chern class of $\left.\mathcal{O}\right|_{\Omega}$ is equal to zero, i.e., $c_{1}\left(\left.\mathcal{O}\right|_{\Omega}\right)=0 ;$ Moreover, the first Chern class of canonical line $c_{1}\left(\left.K^{*}\right|_{M}\right)=0$;

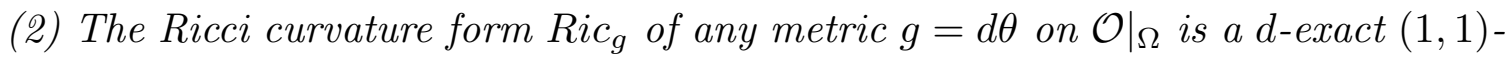
form on $M$. Furthermore, $\operatorname{Ric}(\xi, \bar{\xi})$ is a real number for all $\xi \in T^{(1,0)}(M)$.

Proof. (1) We will use curved version of Kohn-Morrey formula to verify that

$$
c_{1}\left(\left.\mathcal{O}\right|_{\Omega}\right)=0
$$

Recall that the closure $\bar{\Omega}$ of $\Omega$ is compact. Since $V^{2 n}$ is a Stein manifold, there is a strictly pluri-subharmonic function $\phi_{0}$. Let $\phi=\lambda \phi_{0}$ for sufficiently large $\lambda>0$. Using Bochner-Hörmander-Kohn-Morrey formula, we obtain

$$
H^{(p, q)}(\Omega)=0
$$


for all $0<q<n$, (cf. Proposition A.4 of [CaWS, p218]).

It is well-known that, for $\operatorname{dim}_{\mathbb{C}}(\Omega)=n>2$

$$
H^{1}\left(\Omega,\left.\mathcal{O}\right|_{\Omega}\right)=H^{(1,1)}(\Omega)=0
$$

It follows that the first Chern class of $\left.\mathcal{O}\right|_{\Omega}$ is zero.

Choose a Kähler metric $\hat{g}$ on $\Omega$. Then the Ricci curvature form $\hat{\Theta}$ is a $d$-exact $(1,1)$-form.

The classical Kohn-Rossi theory states that any $\bar{\partial}_{b}$-closed $(1,0)$-form on $M=b \Omega$ can be extend to a unique holomorphic $(1,0)$-form on the whole $\Omega$. Thus,

$$
H^{(1,1)}(M)=0
$$

see $[\mathrm{KoR}]$.

It is also known that $c_{1}\left(\left.\mathcal{O}\right|_{M}\right)=c_{1}\left(\left.K^{*}\right|_{M}\right)=0$.

(2) Let $g_{u}$ be the Kähler metric associated with the Kähler form $i \partial \bar{\partial} u$. The corresponding first Chern curvature form $\Theta_{u}$ of the Kähler metric $g_{u}$ is a closed $(1,1)$-form in a neighborhood of $M$ in $V^{2 n}$.

The classical Chern-Weil theory implies that the cohomology class of the first Chern curvature form $\left.\Theta\right|_{M}$ is independent of the choice of the choice of affine connections on $M$.

In fact, if $\theta_{u}=d^{c} u$ and $i \partial \bar{\partial} u>0$, then $d \theta_{u}=d d^{c} u=i \partial \bar{\partial} u>0$ gives rise a Kähler metric in a neighborhood of $M$. For any other $\tilde{\theta}=e^{2 \varphi} \theta$, the Ricci curvature form corresponding to $\tilde{\theta}$ remains to be of type $(1,1)$, see Lemma 2.4 of [L2].

We now recall that a result of Lee [L2].

Proposition 3.3. ([L2, Lemma 6.1, p173-174]) Let $M=b \Omega$ and $\Omega \subset V^{2 n}$ be as in Main Theorem. Suppose that $\tilde{\theta}=e^{2 u} \hat{\theta}$ and $\hat{R} i c$ is the Ricci curvature form corresponding to $\hat{\theta}$. Then $\tilde{\theta}$ is pseudo-Einstein if and only if there is a real solution u satisfying

$$
i \partial_{b} \bar{\partial}_{b} u=\hat{R} i c
$$


Proof. By (6.3) of [L2], the trace-less part of $\tilde{R} i c$ is zero if there is $\varphi$ satisfying

$$
(n+1) i \partial_{b} \bar{\partial}_{b} \varphi=\hat{R} i c
$$

Since $\hat{R} i c$ is a real valued $d$-exact real-valued $(1,1)$-form by Proposition 3.2 above, we can choose $\varphi$ to be real-values as well. (Otherwise, let $v=\frac{1}{2}(\varphi+\bar{\varphi})$ instead).

Proposition 3.4. Let $M=b \Omega$ and $\Omega \subset V^{2 n}$ be as in Main Theorem. Suppose that the Ricci curvature form $\hat{R} i c$ form is a d-exact $(1,1)$-form for the contact 1 -form $\hat{\theta}$. Then there always a real-valued function u satisfying

$$
i \partial_{b} \bar{\partial}_{b} u=\hat{R} i c
$$

Proof. Choose $\sigma$ such that

$$
d \sigma=\hat{R} i c .
$$

Let $\sigma=\sigma^{(0,1)}+\sigma^{(1,0)}+\lambda \theta$, where $\sigma^{(0,1)}$ is $(0,1)$-component of $\sigma$. Since $\hat{R} i c$ is of type $(1,1)$, by $(3.7)$ we have

$$
\bar{\partial}_{b} \sigma^{(0,1)}=0 .
$$

Because $\operatorname{dim}_{\mathbb{C}}(\Omega)>2$, by a Theorem of Kohn that there is complex-valued function $f$ with

$$
i \bar{\partial}_{b} f=\sigma^{(0,1)}
$$

see [CS, Ch9].

It follows that

$$
i \partial_{b} \bar{\partial}_{b} f=\partial \sigma^{(0,1)}=(d \sigma)_{b}=(\hat{R} i c)_{b} .
$$

Since $(\hat{R} i c)_{b}$ is real-valued $(1,1)$-form, choosing $u=\operatorname{Re}\{f\}$, we are done.

We now summarize our result of this section.

Corollary 3.5. Suppose that $\Omega \subset V^{2 n}$ be a compact strictly pseudo-convex domain with smooth boundary in a Stein manifold $V^{2 n}$. Then its boundary $M^{2 n-1}=b \Omega$ admits an intrinsic pseudo-Einstein (i.e., partially Einstein) metric.

Proof. This is a direct consequence of Lemma 3.1 and Propositions 3.2-3.4. 


\section{Estimates for CR Paneitz operators on $M^{3}$}

In the remaining of this paper, we study the so-called CR Paneitz operator

$$
P_{u} f=\triangle_{b}^{2} f+T^{2} f+4 \operatorname{Im} \nabla_{\beta}\left(A^{\alpha \beta} \nabla_{\alpha} f\right),
$$

where $T=J \nabla u$ is the Reeb vector and $A$ is the torsion tensor of the contact form $\theta_{u}$.

When the torsion $A$ vanishes, the formula (4.1) reduces to (1.2).

It remains to verify that CR Paneitz operator $P_{u}$ is a closed operator.

If $\hat{\theta}=e^{\varphi} \theta_{u}$ on $M^{3}$ and $\hat{Q}$ is the corresponding CR $Q$-curvature of the metric associated with the contact form $\hat{\theta}$, then

$$
e^{2 \varphi} \hat{Q}=Q+P_{u} \varphi
$$

see (5.14) of [GG].

Our goal is to show the following result.

Proposition 4.1. Let $\Omega \subset V^{4}$ be an open strictly pseudo-convex domain with compact closure in a Stein manifold $V^{4}$ and let $M^{3}=b \Omega$ be its boundary. Suppose that $g_{u}$ is the Cheng-Yau Einstein metric on $\Omega$ and $\theta_{u}()=.d u(J$.$) is the corresponding$ contact 1-form on $M^{3}$. Then the Paneitz operator $P_{u}$ is closed:

$$
\int_{M^{3}}\left|P_{u} f\right|^{2} \geq c \int_{M^{3}}|f|^{2}
$$

for any real valued function $f \perp k e r P_{u}$, where $c>0$ is a constant independent of $f$.

Remark 4.2: The constant $c$ in Proposition 4.1 depends mostly on the TanakaWebster curvature $R$ and pseudo-hermitian torsion $A_{11}$ of $\left(M^{3}, J, \theta_{u}\right)$ respectively. In fact, the following holds:

$$
\int_{M} 2(P f) f \theta_{u} \wedge d \theta_{u}=\int_{M}\left[3\left(\triangle_{b} f\right)^{2}-\left|H e s s_{b} f\right|^{2}-R\left|\nabla_{b} f\right|^{2}-6 \operatorname{Im}\left\{A_{\overline{11}} f_{1} f_{1}\right\}\right] \theta_{u} \wedge d \theta_{u}
$$


where $\nabla_{b}$ and $H e s s_{b}^{2}$ denotes the sub-gradient and sub-Hessian with respect to $\left(J, \theta_{u}\right)$ respectively, see $[\mathrm{CC}]$.

For the proof of Proposition 4.1, we need some notations.

In what follows, we let $\theta=\theta_{u}$ be the given contact form. The vector $T$ is the characteristic vector in $T(M)$ such that $\theta(T)=1,(d \theta)(T,)=$.0 .

An $(1,0)$-form $\theta^{1} \in \Lambda_{(1,0)}\left(M^{3}\right)$ is called admissible if

$$
\theta^{1}(T)=0, d \theta=i h_{1, \overline{1}} \theta^{1} \wedge \theta^{\overline{1}}
$$

for some hermitian metric function $h_{1, \overline{1}}$.

It is known that

$$
\Delta_{b} f=-f_{\alpha}{ }^{\alpha}-f_{\bar{\alpha}}{ }^{\bar{\alpha}}
$$

and

$$
\square_{b} f=2\left(\bar{\partial}_{b}^{*} \bar{\partial}_{b}+\bar{\partial}_{b} \bar{\partial}_{b}^{*}\right) f=\left(\Delta_{b}+i T\right) f=-2 f_{\bar{\alpha}}{ }^{\bar{\alpha}} .
$$

Inspired by proof of Proposition 3.4 of [L2], we will express the CR Paneitz operator $P$ as a product of several closed operators.

We first consider

$$
\mathcal{L} f=d_{b}^{c} f+\left(\Delta_{b} f\right) \theta
$$

where $\theta$ is the contact 1 -form described above.

Lemma 4.2. Let $M^{3}=b \Omega, \theta, A$ and $\mathcal{L}$ be as above. Suppose that $\Omega \subset V^{4}$ is a strictly pseudo-convex domain in a Stein manifold $V^{4}$ and that $\Omega$ has compact closure. Then $\mathcal{L}$ is a closed operator.

Moreover, one has

$$
d[\mathcal{L} f]=2\left(f_{\overline{1}}{ }^{\overline{1}}{ }_{1}+i A_{11} f^{1}\right) \theta \wedge \theta^{1}+2\left(f_{1}{ }^{1}{ }_{\overline{1}}-i A_{\overline{1} \overline{1}} f^{\overline{1}}\right) \theta \wedge \theta^{\overline{1}} .
$$

Proof. By Theorem 9.4.2 of [CS], both $d_{b}^{c}$ and $\triangle_{b}$ are closed operators for strictly pseudo-convex compact CR-hypersurfaces. Notice that $d_{b}^{c} f \in\left[\Lambda_{(1,0)}\left(M^{3}\right) \oplus \Lambda_{(0,1)}\left(M^{3}\right)\right]$ is always orthogonal to the 1 -form $\left(\Delta_{b} f\right) \theta$. Hence, $\mathcal{L}$ is a closed operator. 
We will use the proof of Proposition 3.4 of [L2].

The $\theta^{1} \wedge \theta^{\overline{1}}$-component of $d[\mathcal{L} f]$ is

$$
i\left[f_{1 \overline{1}}+f_{\overline{1} 1}-\left(f_{1}^{1}+f_{\overline{1}}^{\overline{1}}\right) h_{1 \overline{1}}\right] \theta^{1} \wedge \theta^{\overline{1}}=0 .
$$

On the other hand, the $\theta \wedge \theta^{1}$-component of $d[\mathcal{L} f]$ is

$$
\left[f_{1}{ }^{1}{ }_{1}+f_{\overline{1}}{ }^{\overline{1}}{ }_{1}-i f_{1,0}+i A_{11} f^{1}\right] \theta \wedge \theta^{1} .
$$

It is known (cf. [L2, Section 2]) that

$$
-f_{1}{ }^{1}{ }_{1}+f_{\overline{1}}{ }^{\overline{1}}{ }_{1}+i f_{1,0}+i A_{11} f^{1}=0 .
$$

It follows from (4.4) and (4.5) that the $\theta \wedge \theta^{1}$-component of $d[\mathcal{L} f]$ is equal to

$$
2\left(f_{\overline{1}}{ }^{\overline{1}}{ }_{1}+i A_{11} f^{1}\right) \theta \wedge \theta^{1} .
$$

For the same reason, the $\theta \wedge \theta^{\overline{1}}$-component of $d[\mathcal{L} f]$ is equal to

$$
2\left(f_{1}{ }^{1} \overline{1}-i A_{\overline{1} \overline{1}} f^{\overline{1}}\right) \theta \wedge \theta^{\overline{1}} .
$$

This completes the proof.

Proof of Proposition 4.1. We now consider the composition of operators:

$$
\tilde{P} f=\partial_{b}^{*}\left[(d(\mathcal{L} f))\left\lfloor_{T}\right] .\right.
$$

It follows from that

$$
(d(L f))\left\lfloor_{T}=2\left(f_{\overline{1}}{ }_{1}{ }_{1}+i A_{11} f^{1}\right) \theta^{1}+2\left(f_{1}{ }^{1} \overline{1}-i A_{\overline{1} \overline{1}} f^{\overline{1}}\right) \theta^{\overline{1}} .\right.
$$

We observe that $\partial_{b}^{*}$ acts on $\Lambda_{(1,0)}\left(M^{3}\right)$ trivially. For real valued function $f$, we further consider

$$
\operatorname{Re}[\tilde{P} \circ f]=\operatorname{Re}\left[\bar{\square}_{b} \square_{b} f\right]+4 \operatorname{Im}\left(A_{\overline{11}} f_{1}\right)_{1},
$$

where $\operatorname{Re}\{z\}$ is the real part of complex number of $z$. 
Therefore, it follows from (4.8)-(4.10) that, for real valued function $f$, we have

$$
\operatorname{Re}[\tilde{P} f]=\triangle_{b}^{2} f+T^{2} f+4 \operatorname{Im}\left(A_{\overline{11}} f_{1}\right)_{1}=P f .
$$

Thus, the CR Paneitz operator $P$ satisfies

$$
P f=R e[\tilde{P} f]
$$

where

$$
\tilde{P}=\partial_{b}^{*}\left[(d(\mathcal{L} f))\left\lfloor_{T}\right]\right.
$$

A composition of closed operators remains to be a closed operator.

If $M^{3}=b \Omega$ is a compact strictly pseudo-convex hypersurface in a Stein manifold $V^{4}$, then $\left\{\bar{\partial}_{b}, d, \partial_{b}^{*}, \mathcal{L}\right\}$ are closed operators, by Kohn's $\bar{\partial}_{b}$-theory (cf. [CS, Theorem 9.4.2, p231]). Theorem 9.4.2 of [CS] was stated for $\Omega \subset \mathbb{C}^{2}$, but its proof is applicable to $\Omega$ in all Stein manifolds $V^{4}$ including $\mathbb{C}^{2}$. It is clear that the operator $R e$ is a closed operator. Therefore, $P=\operatorname{Re} \tilde{P}$ is a closed operator as well.

Proof of Main Theorem. Main Theorem now follows from Corollary 2.4, Corollary 3.5 and Proposition 4.1.

Acknowledgement. The first named author would like to thank Professors Alice Chang, Matt Gursky and Paul Yang for many inspiring conversations. In particular, authors are grateful to Matt Gursky for his suggestion of using the notion of "Q-flat metrics". We are very indebted to Professor Jih-Hsin Cheng for pointing out an overlook on the difference between essentially positive operators and closed operators in an earlier version of our paper. Finally, authors would like to thank the referee for his (or her) many suggestions on re-organizing and expositions of results in this paper, including the reference section. 


\section{REFERENCES}

[AV] Andreotti, Aldo; Vesentini, Edoardo, Carleman estimates for the Laplace-Beltrami equation on complex manifolds, Inst. Hautes Etudes Sci. Publ. 25 (1965), 80-130.

[Br] Brendle, S., Global existence and convergence for a higher order flow in conformal geometry, Ann. of Math. 158 no.1 (2003), 323-343.

[CaWS] Cao, J., M. Shaw and Wang, L, Estimates for the $\bar{\partial}$-Neumann problem and nonexistence of $C^{2}$ Levi-flat hypersurfaces in $\mathbb{C P}^{n}$, Math. Zeit 248 (2004), 183-221.

[Ch] Chang, A, Conformal invariants and partial differential equations, Bull. Amer. Math. Soc. (N.S.) 42 no. 3 (2005), 365-393.

[CC] Chang, Shu-Cheng and Chiu, Hung-Lin, On the estimate of first eigenvalue of a sublaplacian on a pseudo-hermitian 3-manifold, to appear in "Pacific Journal of Mathematics".

[CCC] Chang, Shu-Cheng, Cheng, J. H. and Chiu, Hung-Lin, The Fourth-order Q-curvature flow on a CR 3-manifold, to appear in Indiana University Math Journal.

[CS] Chen, S.-C. and Shaw, M.-C., Partial Differential Equations in Several Complex Variables, American Math. Society-International Press, Studies in Advanced Mathematics, Volume 19, Providence, R.I., 2001.

[CY] Cheng, Shiu-Yuen and Yau, S-T.:, On the existence of a complete Kähler metric on noncompact complex manifolds and the regularity of Fefferman's equation, Comm. Pure Appl. Math. 33, no. 4 (1980), 507-544.

[DF] Diederich, K. and Fornaess, J. E., Pseudoconvex domains: Bounded strictly plurisubharmonic exhaustion functions, Invent. Math. 39 (1977), 129-144.

[DT] Dragomir, S. and Tomassini, G., Differential geometry and analysis on CR manifolds, Birkhäuser Verlag, c2006, Progress in mathematics ; vol 246, Boston, 2006.

[F1] Fefferman, C, The Bergman kernel and biholomorphic mappings of pseudoconvex domains, Invent. Math. 26 (1974), 1-65.

[F2] Fefferman, C, Monge-Ampére equations, the Bergman kernel, and geometry of pseudoconvex domains, Correction, Ann. of Math. (2) 104 (1976), no. 2, 393-394., Ann. of Math 103 (1976), 395-416.

[FG1] Fefferman, C. and Graham, C. Robin, Conformal invariants, The mathematical heritage of Élie Cartan (Lyon, 1984). Astérisque 1985, Numero Hors Serie, 139-151.

[FG2] Fefferman, C. and Graham, C. Robin, Q-curvature and Poincaré metrics, Math. Res. Lett 9 (2002), 139-151.

[FH] Fefferman, C. and Hirachi, K., Ambient metric construction of Q-curvature in conformal and CR geometries, Math. Res. Lett 10, no 5-6 (2003), 819-831.

[GG] Gover, A. Rod and Graham, C. Robin, CR Invariant powers of the sub-Laplacian, J. Reine Angew. Math. 583 (2005), 1-27.

[Hö1] Hörmander, L., $L^{2}$ estimates and existence theorems for the $\bar{\partial}$-operators, Acta Math. 113 (1965), 89-152.

[Hö2] Hörmander, L., An introduction to complex analysis in several complex variables, Third Edition, Van Norstand, Princeton, 1990.

[Ko1] Kohn, J, Global regularity for $\bar{\partial}$ on weakly pseudo-convex manifolds, Trans. Amer. Math. Soc. 181 (1973), 273-292.

[Ko2] Kohn, J, The range of the tangential Cauchy-Riemann operator, Duke Math. J. 53, no. 2 (1986), 525-545.

[Ko3] Kohn, J, Superlogarithmic estimates on pseudoconvex domains and CR manifolds, Annals of Math. 155 No.2 (2002), 213-348.

[KoR] Kohn, J. and Rossi, H., On the extension of holomorphic functions from the boundary of a complex manifold, Annals of Math. 81 (1965), 451-472. 
[L1] Lee, J, The Fefferman metric and pseudo-Hermitian invariants, Trans. Amer. Math. Soc. 296, no. 1 (1986), 411-429.

[L2] Lee, J, Pseudo-Einstein structures on CR manifolds, Amer. J. Math. 110, no. 1 (1988), 157-178.

[LL] Li, Song-Ying and Luk, Hing-Sun, An explicit formula for the Webster pseudo Ricci curvature on real hypersurfaces and its application for characterizing balls in $\mathbb{C}^{n}$, Communications in Analysis and Geometry 14, no. 4 (2006), 673-701.

[Mi] Milnor, J. W., Characteristic classes, Princeton University Press, Princeton, N. J., 1974.

[MY] Mok, N. and Yau, S. T.:, Completeness of the Kähler-Einstein metric on bounded domains and the characterization of domains of holomorphy by curvature conditions, The mathematical heritage of Henri Poincaré, Part 1 (Bloomington, Ind., 1980), 4159, Proc. Sympos. Pure Math., 39, Amer. Math. Soc., Providence, RI, 1983., 41-59.

[Siu] Siu, Y. T., Complex-analyticity of harmonic maps, vanishing and Lefschetz theorems, J. Diff. Geom. 17 (1982), 55-138.

[Ta1] Tanaka, N, On the pseudo-conformal geometry of hypersurfaces of the space of $n$ complex variables, J. Math Soc. Japan. 14 (1962), 397-429.

[Ta2] Tanaka, N, A differential geometric study on strongly pseudo-convex manifolds, Lectures in Mathematics, vol 9, Department of Mathematics, Kyoto University, Kinokunia Book-Store Co. Ltd, Tokyo, Japan, 2001.

[Web] Webster, S, Pseudo-hermitian structures on a real hypersurface, J. Diff. Geom. 13 (1978), 25-41.

Mathematics Department, University of Notre Dame, Notre Dame, IN 46556

E-mail address: jcao@nd.edu

Department of Mathematics, National Tsing Hua University, Hsinchu 30013, TaiWAN, R. O. C.

E-mail address: scchang@math.nthu.edu.tw 